\title{
Impact of the COVID-19 pandemic in Radiopharmacy Activities: Preliminary report: first international survey
}

\author{
Fatma Al-Saeedi ${ }^{1}$ \\ ${ }^{1}$ Kuwait University
}

July 23, 2020

\begin{abstract}
Rationale, aims, and objectives: The coronavirus pandemic has disrupted normal activities across the world, from economy to socialization, and claiming hundreds of thousands of lives. The activities in radiopharmacy laboratories across the globe have been affected significantly. This survey was conducted to assess the effect of COVID-19 on radiopharmacy and activities going on in the laboratories around the world. This was meant to undertake a preliminary record of the continuing survey. Methods: A three-sectioned questionnaire with 12 questions was distributed to the professional working on radiopharmacy laboratories. The sections included demographic information, the effect of the pandemic on the regular operation on a radiopharmacy, and the measures taken to prevent the spread of the virus among the laboratory staff. Random sampling approach was used to select 100 respondents, including staff and radiochemists, across different parts of the world. Results: The survey demonstrated that the pandemic hugely impacted activities in a radiopharmacy laboratory. $98 \%$ of the respondents reported major disruptions in the normal schedule in the laboratory. Even though some laboratories continued working normally, strict measures were put in place to prevent the spread of the virus among the staff. In many radiopharmacy laboratories, diagnostic procedures and tests quantitatively decreased. The survey reported positron emission radiopharmaceutical fluorodeoxyglucose F-18 (F18-FDG) as the frequently organ imaging radiopharmaceutical during the pandemic. Conclusions: The survey showed that COVID-19 has negatively impacted activities in a radiopharmacy laboratory, consequently affecting research activities.
\end{abstract}

\section{Introduction}

COVID-19 is a contagious disease caused by the coronavirus. ${ }^{1-3}$ The virus is transmitted through droplets produced when an infected individual exhales, sneezes, or coughs. One gets infected by breathing in the virus when within close distance with an infected individual or by touching a contaminated surface and then your mouth, nose, or eyes. The pandemic has claimed many lives across the globe and brought the world economy to a standstill. The medical sector has significantly suffered as a result of the pandemic. Currently, the virus has no cure, but scientists all over the world are working day and night to find a vaccine. ${ }^{4-8}$ People are encouraged to take preventive measures to avoid the spread of the virus, including the stay at home calls, social distancing, wearing masks, and regular washing of hands. ${ }^{9-11}$

Apart from the hurting and painful impact on the economy and the daily number of deaths across the world, COVID-19 is also impacting the activities in medical centers. As observed by this study, the pandemic has dramatically affected the activities in radiopharmacy laboratories around the world. This survey aimed to assess the impact of the coronavirus pandemic on activities inside radiopharmacy laboratories globally. The survey looked into the activities going on in the laboratories and what has been affected by the pandemic.

Methods: A Google web-based questionnaire with 12 multiple choice questions were designed to gather data regarding the activities in radiopharmacy laboratories and how COVID-19 has affected their operations.

Survey Invitations: The respondents were requested to take part in the survey through email invitations. Using Google Form Documents, the questionnaire was delivered to the individual respondents working in 
various radiopharmacy laboratories across the globe using a link and attachments. A reminder was sent to those who did not show a response, asking them to take part in the survey. The survey was voluntary, and no one was coerced into participating against their will.

Sections of the Questionnaire: The 12 point questionnaire was designed and delivered to 100 professional working radiopharmacy laboratories in different parts of the world to get a picture of the impact of COVID19 on operations within the facilities. The questionnaire was divided into three sections: (1) demographic information, (2) effects of the pandemic on the activities of the respective radiopharmacy lab, and (3) measures taken to prevent or limit the spread of the virus among the staff.

Data Analysis: Results of the survey were analyzed using Microsoft Excel spreadsheets. Direct responses received from the emails and free texts were also summed up and incorporated in the results.

Results: Demographic data - the survey respondents were organized in accordance with their names, country, age, and profession. The grouping was also based on individual organization, the institution of work, center, and department. One hundred participants randomly chosen from different countries, including United Kingdom (UK), Kingdome of Saudi Arabia, USA, India, Greece, Italy, Slovenia, Scotland, South Africa, and Kuwait, were used in the survey. However, most of them were from the UK.

Around $81.4 \%$ of the respondents were aged between 40 and $60,10 \%$ between 60 and 80 , and $8.2 \%$ between 20 40 years. Different health professionals were randomly sampled. $1 \%$ were radiochemists, $1 \%$ from the teaching faculty, $1 \%$ were scientists, $4 \%$ medical physicians, $11.5 \%$ health administrators, and $80.5 \%$ technologists (Fig.1).

The pandemic affected scheduling criteria, with most of the staff encouraged to stay at home or observe the social distance. $1 \%$ of the respondents reported going about their normal operations but had strict measures to prevent the spread of the virus. In a large number of radiopharmacy labs, diagnostic imaging tests quantitatively reduced. Certain imaging centers reduced their test procedures to almost half (18.2\%). Others reported delay in performing the tests (5\%).

It was also reported that COVID-19 had changed procedures for radiopharmaceutical preparations in NM departments by interfering with their pattern of work. F18-FDG (Fig.2) was reported as the frequently used organ imaging pharmaceutical during the pandemic $(77 \%)$. Other organs include bone (13\%), heart (1\%), lungs $(3 \%)$, and others $(6 \%)$.

The result of the survey also shows that COVID-19 has changed the purchase of pharmaceuticals by $96.9 \%$. Most of the laboratories have taken COVID-19 seriously and implemented the WHO recommendations and regulations on preventing the spread of the virus. $99 \%$ of the respondents report strict measures including hand hygiene, social distancing, wearing PPEs, and reduction in the number of professionals allowed in a laboratory.

\section{Discussion}

The results of this survey were in agreement with studies emphasizing the role of F18-FDG during COVID19. ${ }^{12-15}$ Some studies reported the need for lung, bone, and inflammation -related radiopharmaceuticals. $16-17$

The results are in agreement with many studies reported many changes in the transport of medical radioisotopes, purchasing, and preparations of radiopharmaceuticals. The results are in agreement with the fact that the supply, the availability of the most vital medical radioisotopes used in radiopharmacy of nuclear medicine and in research are delayed or stopped due to the current lockdown, extended border controls, cancelation of most of commercial passenger flights, increments in the cargo costs. ${ }^{18-19}$ The results showed that it is a must and yes there is intention to develop new radiopharmaceuticals to detect the impact and to help to know the cause of COVID-19 pandemic and confine it.

The COVID-19 pandemic has forced many organizations back to the drawing board to redesign their operations and strategy. It is clear that the pandemic is continuing to have a huge impact on the medical 
sector by affecting normal operations. It has created a relatively inconvenient environment of operations within radiopharmacy labs across the globe due to strict guidelines that must be followed to avoid the spread of the virus. As the rate of infection continues to increase each day, the number of deaths also increases. The increased infection rate has caused a strain in medical facilities. Access and demand for medical and laboratory equipment such as radioisotopes and the preparation of radiopharmaceuticals have drastically changed. Medical professionals must conform to the new ways of life and accept that things are different in order to prevent the spread of the virus. ${ }^{20-21}$

\section{Conclusion}

The exploratory survey report shows that COVID-19 is negatively impacting the normal operation of radiopharmacy labs, consequently affecting the research works of NM. The study survey provided a new perspective of handling future pandemics and unearths the efforts of research professionals to find a cure and radiopharmaceuticals to do away with the virus. The regulations recommended by WHO and government and regular information or updates regarding COVID-19 have been widely used. So far, the coronavirus has no cure, and people must rely on preventive measures such as washing hands, social distancing, stay at home calls, and wearing masks.

\section{Data and material availability}

Upon a reasonable request, the data supporting the findings of this study can be given by the author.

\section{Competing interest}

There are no conflicts of interest to disclose.

\section{Funding}

None.

\section{Author's contributions}

The author took part in the conception, design, material preparation, collection, and analysis. The author wrote the initial draft of this report, read and approved the final copy, and wrote the cover letter.

\section{Acknowledgment}

My gratitude to all those who took their time to respond to the questionnaire survey and for their advice, comment, and support.

\section{References}

1. Xu XW, Wu XX, Jiang XG, Xu KJ, Ying LJ, Ma CL, Li SB, Wang HY, Zhang S, Gao HN, Sheng JF, Cai HL, Qiu YQ, Li LJ. Clinical findings in a group of patients infected with the 2019 novel coronavirus (SARS-Cov-2) outside of Wuhan, China: retrospective case series. Version 2. BMJ. 2020;368:m606. doi: 10.1136/bmj.m606.

2. Huang C, Wang Y, Li X, Ren L, Zhao J, Hu Y, Zhang L, Fan G, Xu J, Gu X, Cheng Z, Yu T, Xia J, Wei Y, Wu W, Xie X, Yin W, Li H, Liu M, Xiao Y, Gao H, Guo L, Xie J, Wang G, Jiang R, Gao Z, Jin Q, Wang J, Cao B. Clinical features of patients infected with 2019 novel coronavirus in Wuhan, China. Lancet. 2020;395(10223):497-506. doi: 10.1016/S0140-6736(20)30183-5.

3. Chen N, Zhou M, Dong X, Qu J, Gong F, Han Y, Qiu Y, Wang J, Liu Y, Wei Y, Xia J, Yu T, Zhang $\mathrm{X}$, Zhang L. Epidemiological and clinical characteristics of 99 cases of 2019 novel coronavirus pneumonia in Wuhan, China: a descriptive study. Lancet. 2020;395(10223):507-513. doi: 10.1016/S01406736(20)30211-7.

4. Ahn DG, Shin HJ, Kim MH, et al. Current Status of Epidemiology, Diagnosis, Therapeutics, and Vaccines for Novel Coronavirus Disease 2019 (COVID-19). J Microbiol Biotechnol. 2020;30(3):313324. doi:10.4014/jmb.2003.03011. 
5. Raptis CA, Hammer MM, Short RG, Shah A, Bhalla S, Bierhals AJ, et al. Chest CT and coronavirus disease (COVID-19): a critical review of the literature to date. AJR Am J Roentgenol. 2020;16:1-4.

6. Guedj E, Verger A, Cammilleri S. PET imaging of COVID-19: the target and the number. Eur J Nucl Med Mol Imaging 2020; 17.

7. Juengling FD, Maldonado A, Wuest F, Schindler TH. The role of nuclear medicine for COVID-19 time to act now. J Nucl Med 2020; 17.

8. Mboowa G. Current and emerging diagnostic tests available for the novel COVID-19 global pandemic. AAS Open Res. 2020;3:8. doi:10.12688/aasopenres.13059.1.

9. Editorial Nature. Coronavirus: three things all governments and their science advisers must do now. Nature. 2020;579(7799):319-320.

10. Peters A. The global proliferation of high-containment biological laboratories: understanding the phenomenon and its implications. Rev Sci Tech. 2018;37(3):857-883.

11. Buscombe JR, Notghi A, Croasdale J, PanditM,O'Brien J, Graham R, Council and Officers of British Nuclear Medicine Society, et al. COVID-19: guidance for infection prevention and control in nuclear medicine. Nucl Med Commun. 2020.

12. Treglia G. The role of (18)F-FDG PET for COVID-19 infection: myth versus reality. Clin Transl Imaging. 2020;30:1-2.

13. Lütje S, Marinova M, Kütting D, Attenberger U, Essler M, Bundschuh RA. Nuclear medicine in SARSCoV-2 pandemia: 18F-FDG-PET/CT to visualize COVID-19. Nuklearmedizin 2020; 7.

14. Scarlattei M, Baldari G, Silva M, et al. Unknown SARS-CoV-2 pneumonia detected by PET/CT in patients with cancer. Tumori. 2020;300891620935983. doi:10.1177/0300891620935983.

15. Qin C, Liu F, Yen T, Lan X. 18F-FDG PET/CT findings of COVID-19: a series of four highly suspected cases. Eur J Nucl Med Mol Imaging. 2020. https://doi.org/10.1007/s00259-020-04734-w.

16. Zuckier LS, Gordon SR. COVID-19 in the nuclear medicine department be prepared for ventilation scans as well! Nucl Med Commun. 2020;41(5):494-5.

17. Setti L, Kirienko M, Dalto SC, Bonacina M, Bombardieri E. FDG-PET/CT findings highly suspicious for COVID-19 in an Italian case series of asymptomatic patients. Eur J Nucl Med Mol Imaging. 2020;47(7):1649-1656. doi:10.1007/s00259-020-04819-6.

18. Freudenberg LS, Dittmer U, Herrmann K. Impact of COVID-19 on Nuclear Medicine in Germany, Austria and Switzerland: An International Survey in April 2020. Nuklearmedizin. 2020; doi: 10.1055/a1163-3096.

19. Chatzipavlidou V. A new era for nuclear medicine. Hell J Nucl Med. 2020;23(1):2-3. doi:10.1967/s002449912013.

20. LamWW, Loke KS,WongWY, Ng DC. Facing a disruptive threat: how can a nuclear medicine service be prepared for the coronavirus outbreak 2020? Eur J Nucl Med Mol Imaging. 2020.

21. Zhang, X., Shao, F. \& Lan, X. Suggestions for safety and protection control in Department of Nuclear Medicine during the outbreak of COVID-19. Eur J Nucl Med Mol Imaging 2020; 47, 1632-1633.

\section{Figure legends}

Fig. 1. Profession of the Respondents

Fig. 2. Imaging Pharmaceuticals

\section{Hosted file}

Fig1-2_JEVALCLINpRCT.ppt available at https://authorea.com/users/345425/articles/471659impact-of-the-covid-19-pandemic-in-radiopharmacy-activities-preliminary-report-firstinternational-survey 